\title{
Distribución de la silvicultura en la cuenca hidrográfica del rio Yaguarón (Brasil-Uruguay) basado en métricas del paisaje
}

\author{
Distribuição da silvicultura na bacia do Rio Jaguarão (Brasil- \\ Uruguai) com base na métrica de paisagem
}

\author{
Distribution of Forestry in the Jaguarão River Basin (Brazil- \\ Uruguay) based on Landscape Metrics
}

\author{
Maribel Olaya Betancourt \\ lilium.umi@gmail.com \\ Universidade de Brasília, DF \\ Valdir Adilson Steinke \\ valdirsteinke@gmail.com \\ Universidade de Brasilia, DF
}

\begin{abstract}
Resumen: Este trabajo presenta un análisis de la distribución de la silvicultura en la cuenca hidrográfica del rio Yaguarón localizada en la frontera Brasil y Uruguay. Para esto se realizó un mapeo de fragmentos de silvicultura en el área de estudio en escala 1:20.000, obteniéndose 6.730 fragmentos de silvicultura. Los fragmentos de silvicultura fueron agrupados por clases de áreas y clases de vegetación de la zona de estudio para mejor análisis de las métricas. También se dividió la cuenca hidrográfica en trechos superior, medio e inferior. Los datos obtenidos mostraron homogeneidad entre los fragmentos de silvicultura, y que su distribución es influenciada principalmente por el relieve. Además, se comprobó que la cantidad de fragmentos pequeños de silvicultura en la cuenca hidrográfica responden históricamente a procesos culturales más que a procesos económicos actuales.
\end{abstract}

Palabras-Claves: foresta, Bioma Pampa, cuenca hidrográfica transfronteriza, métricas del paisaje.

Resumo: Este trabalho apresenta uma análise da distribuição da silvicultura na bacia do rio Jaguarão, localizado na fronteira entre Brasil e Uruguai. Para o mapeamento dos fragmentos florestais foi conduzido um estudo, na escala 1:20.000, obtendo-se 6.730 fragmentos de silvicultura. Os fragmentos florestais foram agrupados por classes de área e por tipos de vegetação da área de estudo para uma análise mais completa das métricas. A bacia hidrográfica foi dividida em trecho superior, médio e inferior. Os dados obtidos demonstraram homogeneidade entre fragmentos silvicultura, e a sua distribuição espacial sofre forte influência do relevo. Além disso verificou-se que o número significativo de pequenos fragmentos de florestas na bacia responde por processos culturais históricos, enquanto que os fragmentos maiores estão associados aos processos econômicos mais recentes.

Palavras-chave: floresta, Bioma Pampa, bacia hidrográfica transfronteiriça, métricas de paisagem. 
Abstract: This work presents an analysis of the distribution of forestry in the Jaguarão river basin, located on the border between Brazil and Uruguay. For the mapping of forest fragments, it was conducted a study, in the scale 1:20,000, obtaining 6,730 silvicultural fragments grouped by area classes and vegetation types for a more complete analysis of the metrics. The watershed was divided into upper, middle and lower stretches. The obtained data demonstrated homogeneity among silvicultural fragments, and their spatial distribution suffers strong influence of the relief. In addition, it has been found that the significant number of small forest fragments in the basin responds by historical cultural processes, whereas larger fragments are associated with the more recent economic processes.

Keywords: Forest, Pampa Biome, Cross-border river basin, Landscape Metrics.

\section{INTRODUCCÍON}

La ecología del paisaje nació a mediados de siglo XX en Europa central y occidental impulsada principalmente por el geógrafo Car Troll, con el objetivo de ser una ciencia integradora de disciplinas sociales, geofísicas, y biológicas buscando una comprensión global del paisaje, principalmente cultural, y de ordenamiento territorial. En los años 80 la ecología del paisaje es introducida en los Estados Unidos por biogeógrafos y ecólogos, influenciada por la ecología de ecosistemas y por el modelaje y análisis espacial, enfatizando en la conservación de la biodiversidad y el manejo de los recursos naturales (METZGER, 2006).

Actualmente entre los principales objetivos de la ecología del paisaje, está el estudio de los efectos entre el patrón espacial y los procesos ecológicos que se manifiestan a escala del paisaje, como consecuencia de la heterogeneidad espacial a través de las escalas que rigen las comunidades y poblaciones (FORMAN; GODRÓN, 1981; FORMAN, 1995; PICKETT; CADENASSO, 1995; MATTEUCCI; MATTEUCCI; BUZAI, 1998; AYAD, 2005; IRASTORZA V., 2006), como de los cambios en el paisaje causado por procesos naturales o acciones humanas que resultan en fragmentación (FORMAN, 1995).

La heterogeneidad en un paisaje se debe principalmente a tres propiedades básicas: la estructura (organización espacial), función (interacciones entre los elementos) y cambio (dinámica o transformación, en la estructura y función a lo largo del tiempo), donde su identificación y medida dependerá de la escala espacial de observación (FORMAN; GODRÓN, 1981; MEENTEMEYER; BOX, 1987; MATTEUCCI; MATTEUCCI; BUZAI, 1998).

En la ecología del paisaje la cuantificación de la estructura es considerada una herramienta fundamental para la descripción, interpretación y análisis de la configuración espacial y las asociaciones entre padrón y procesos, reduciendo la complejidad del paisaje a un conjunto de valores numéricos, denominados índices o métricas del paisaje (METZGER, 2006), siendo fácilmente analizadas y manipuladas a partir de técnicas de teledetección y de Sistemas de Información Geográficos SIGs (FARINA, 2008). 
Las métricas del paisaje se dividen en índices de composición e índices de disposición; los índices de composición se relacionan con las unidades del paisaje, indicando la dominancia espacial de los fragmentos y los índices de disposición cuantifican la organización espacial de cada mancha en términos de grado de fragmentación, conectividad y forma de los fragmentos (METZGER, 2006).

Actualmente existe una gran variedad de métricas disponibles para análisis del paisaje, cada una desarrollada para resolver situaciones específicas en las investigaciones (AYAD, 2005), de este modo, en la mayoría de las investigaciones donde se aplican métricas del paisaje, son usadas solo un determinado número de métricas, siendo suficientes para conectarse a los criterios ecológicos más eficaces y relevantes para su análisis (LANG; TIEDE, 2003).

El objetivo de este trabajo fue observar y analizar la distribución de la silvicultura de árboles de los géneros Pinus y Eucalyptus en la cuenca transfronteriza del rio Yaguarón entre Brasil y Uruguay, usando métricas del paisaje, con la finalidad de identificar cambios e impactos generados por dichos cultivos en esta cuenca hidrográfica.

Algunos autores resaltan, que en áreas estratégicas de frontera, las políticas económicas de desenvolvimiento regional tanto en Brasil como en Uruguay, han apoyado iniciativas de implementación de silvicultura como estrategia de desarrollo económico, causando grandes transformaciones en la conformación social, espaciales y ambiental y afectando el cotidiano de los pobladores adaptados a las características geográficas naturales del Bioma Pampa, inserido en esas zonas fronterizas (DIAZ; TESON; GARCIA, 2006; CHELOTTI, 2010; RÓTULO; DAMIANI, 2010; CLEMENTE B., 2014).

\section{MATERIALES Y MÉTODOS}

\section{Localización geográfica y caracterización del área de estudio}

La cuenca hidrográfica transfronteriza del rio Yaguarón se sitúa al Sudoeste del estado de Rio Grande del Sur (Brasil) y al Nordeste del departamento de Cerro Largo

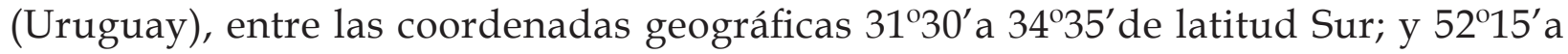
$55^{\circ} 15^{\prime}$ de longitud Oeste. Hace parte de la red hidrográfica que conforma la cuenca hidrográfica binacional de la Laguna Merín (Fig. 1). Tiene un área de aproximadamente $8.188 \mathrm{~km}^{2}$, de los cuales $78 \%$ se encuentra en territorio Brasileño y $22 \%$ en territorio Uruguayo (FEPAM, 2013). 
Figura 1: Localización de la cuenca hidrográfica del rio Yaguarón en la frontera entre Uruguay (UY) y Rio Grande do Sul, Brasil (BR-RS) y al interior de la Laguna Merín.

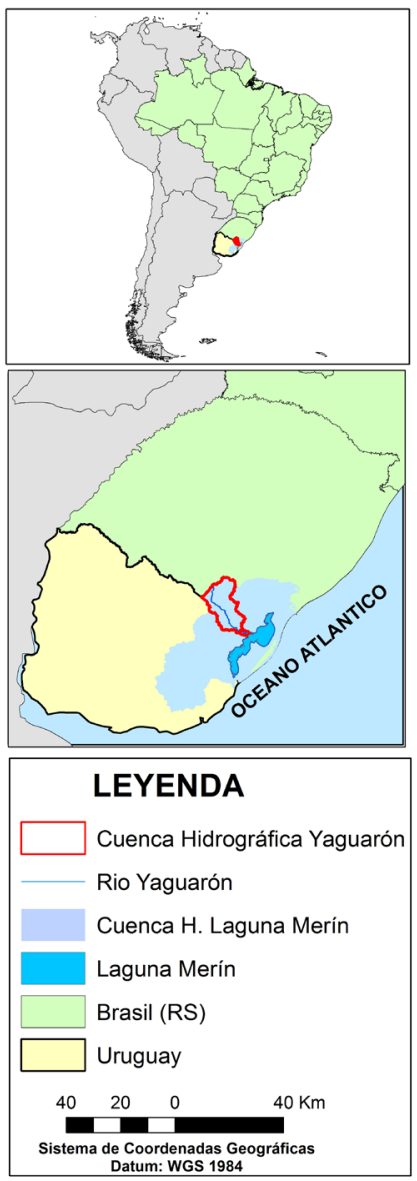

Coordenadas Geográficas
Datum: WGS 1984

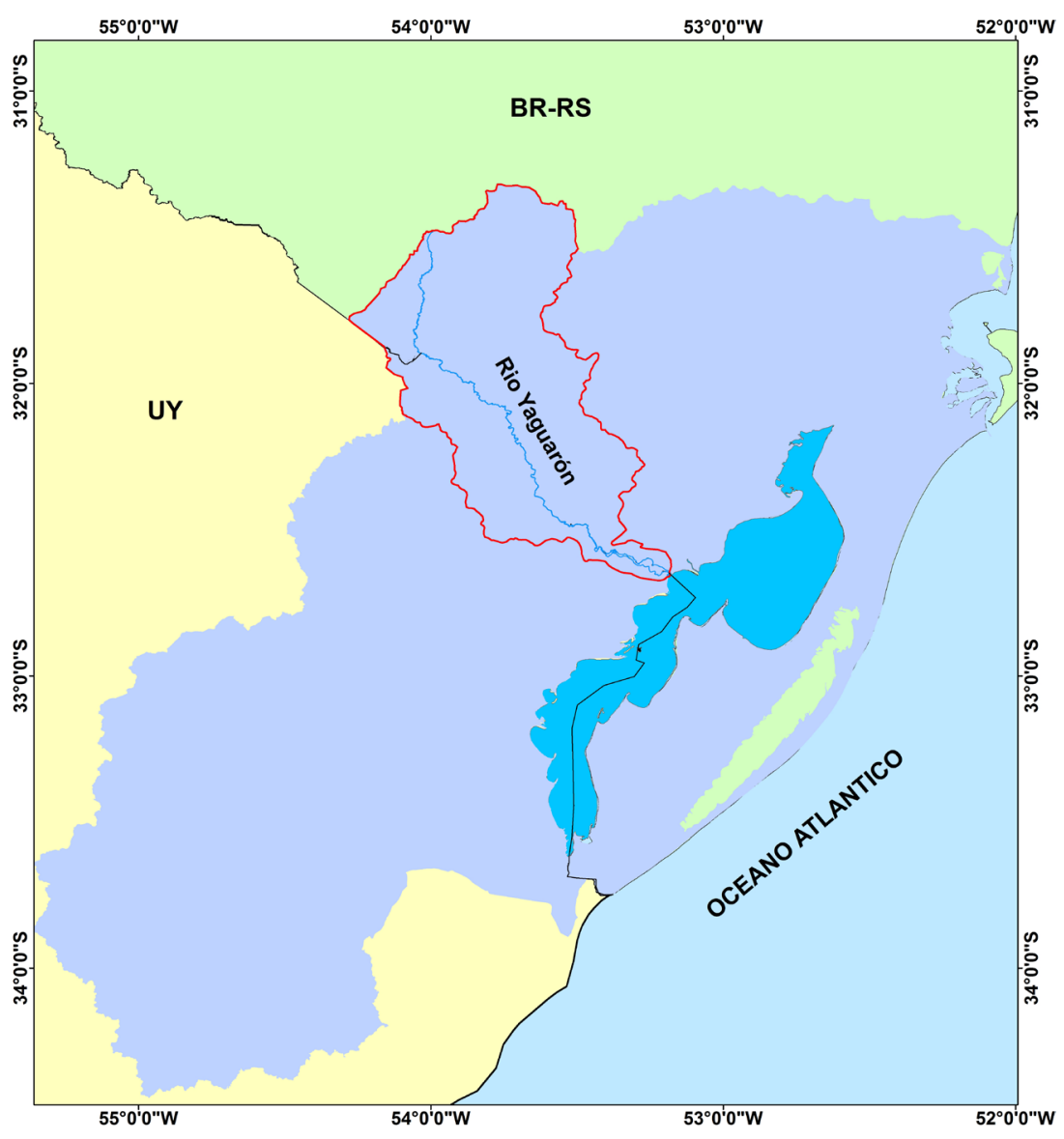

Fuente: autores

El nacimiento del rio Yaguarón (Jaguarão) está localizado en la ciudad de Bagé Brasil, recorriendo para sudeste hasta desembocar en la Laguna Merín. Del lado brasileño se destacan los Arroyos: Candiota, Jaguarão Chico, São Jose, Butiá, do Bote, do Meio, do Telho, (FEPAM, 2013). En Uruguay el rio Yaguarón se caracteriza por ser el rio más caudaloso del departamento de Cerro Largo, sus principales afluentes son los Arroyos de las Cañas, Sarandí de Barcelo, y Sarandí (PEREZ ÁLVAREZ, 2014).

La cuenca hidrográfica presento durante un periodo de análisis de 30 años una precipitación y evaporación media anual de 1.245,10mm y 1.303,65mm respectivamente. El caudal promedio del rio Yaguarón es de $86,3 \mathrm{~m}^{3} / \mathrm{s}$ y su descarga específica es 0,0182 $\mathrm{m}^{3} / \mathrm{km}^{2}$. El Pampa y sus paisaje fisiográfico caracterizan a la cuenca hidrográfica del rio Yaguarón destacando relieves que van desde afloramientos rocosos a relieves planos o poco inclinados con elevaciones desde los 100 a 400 metros sobre el nivel del mar (VERDUM; BASSO; SUERTEGARAY, 2004).

Según Sombroek et al. (1970) la cuenca hidrográfica del rio Yaguarón está inserida en los ambientes paisajísticos de la Laguna Merín correspondientes a las colinas gondwânicas; sierras (rocosas y no rocosas); planicies altas y fluviales; colinas y lomadas sedimentarias; colinas y lomadas cristalinas; planicies bajas y bañados fluviales. 
La vegetación entre el sur del Brasil y Uruguay es definida por su continuidad geológica manifestada en las características geomorfológicas, fisiográficas y edáficas de los territorios, viabilizando las conexiones entre los tipos de vegetación de Rio Grande del Sur con la vegetación del Uruguay (CRACCO et al., 2005). La cuenca hidrográfica es constituida por un conjunto de vegetación campestre, relativamente uniforme donde predomina la cobertura vegetal de tipo estepa o de praderas, seguida de vegetación de tipo Floresta Estacional Decidual y en menor expresión la vegetación de Savanas Estepicas y la vegetación de Formações Pioneiras de Influência Fluvial ${ }^{1}$ (HASENACK; CORDEIRO, 2006; ACHKAR; DOMINGUEZ; PESCE, 2012; MARCHESI; FAROPPA; ANNALA, 2005; CAL et al., 2008).

En Brasil, la cuenca hidrográfica incluye, total o parcialmente, nueve municipios: Aceguá, Arroio Grande, Bagé, Candiota, Harval, Hulha Negra, Pedras Altas, Pinheiro Machado y Jaguarão (FEPAM, 2013), la población total del área de la cuenca en Brasil es de 45.415 habitantes. Ya en el área abarcada en Uruguay la cuenca está sobre los municipios de Aceguá con 1.511 habitantes, Isidoro Noblía con 2.331 y Rio Branco, con 14.604 habitantes (INE, 2013).

La estructura productiva de la cuenca hidrográfica está basada en el sector agropecuario, donde se constata el desarrollo de polos tecnológicos unidos a la industrialización de productos agropecuarios. Del lado uruguayo se destaca, la producción de arroz, sorgo, cebada, la producción forestal, producción de carne y productos lácteos (ACHKAR DOMINGUEZ; PESCE, 2012; SERRENTINO B., 2013); del lado brasileño de la cuenca hidrográfica se destacan las culturas de arroz, soya, trigo, sorgo, cebada y maíz, la silvicultura, la fruticultura de uva, sandia, naranja y melocotón además la ganadería de corte y lechera, la ovinocultura y en menor representatividad la acuicultura y producción de miel de abejas (IBGE, 2015). Se destacan también actividades mineras de carbón y cal en el municipio de Candiota (CRM, 2014). Como polo dinamizador económico y turístico, se encuentran los free shops en las ciudades gemelas de Aceguá- Aceguá y Rio Branco-Jaguarão. A los dos lados de la frontera entre Brasil y Uruguay, hay un alto porcentaje de hogares con necesidades básicas insatisfechas y condiciones de vida precarias para la población (CLEMENTE B., 2014; RÓTULO; DAMIANI, 2010).

\section{Procedimientos Metodológicos}

A continuación se presenta en la Figura 2 el organización representativa de los procedimientos metodológicos seguidos para este artículo.

1 En Uruguay, las coberturas vegetales relacionadas al área de estudio son denominadas: praderas, mosaicos de praderas con vegetación arbórea de parques y vegetación de pajonales (ACHKAR DOMINGUEZ; PESCE, 2012). Para este trabajo se adoptó la nomenclatura brasileña propuesta por el Instituto Brasileiro de Geografía e Estatística (IBGE) en el año 2012. 
Figura 2: Diagrama de flujo de los procedimientos metodológicos.

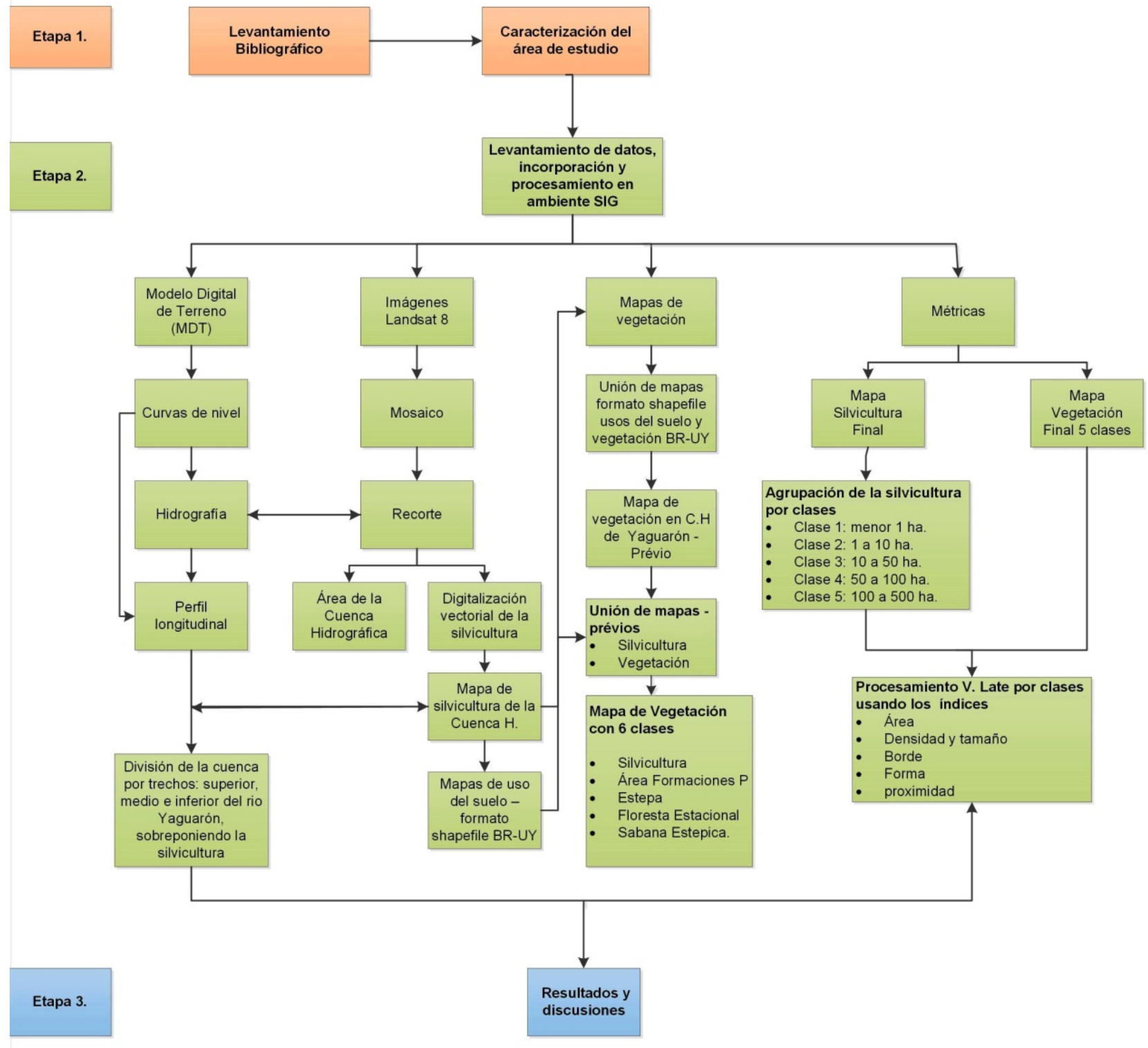

Fuente: autores

La primera etapa representa el levantamiento de informaciones por medio de la bibliografía, sobre la ecología del paisaje y las métricas del paisaje, y las informaciones correspondientes a la caracterización del área de estudio como hidrografía, geología y relieve, vegetación y aspectos económicos y sociales. La segunda etapa representa el orden de recolección, obtención y procesamiento de datos a través de software de sistemas de información geográfico SIG ArcGis 10.1 hasta llegar al procesamiento de las métricas del paisaje. La tercera etapa hace referencia a la obtención de resultados y discusiones. 


\section{Procesamiento de métricas del paisaje}

Se llevó a cabo la aplicación de las métricas del paisaje correspondientes a los fragmentos de silvicultura en primer lugar a nivel de clases agrupados por hectáreas, buscando analizar la espacialización y tamaño de los fragmentos, en segundo lugar, a nivel de paisaje, para analizar las clases de vegetación de la zona de estudio y así observar el impacto de la silvicultura sobre las clases de vegetación natural. Finalmente, para los trechos superior, medio e inferior de la cuenca hidrográfica los fragmentos de la silvicultura fueron analizados por clases de tamaño para observar su dinámica en cada trecho. Para esto se utilizó la extensión gratuita V-Late 2.0 beta (Vector based Landscape Analysis Tools Extension) para ArcGis 10.1. De acurdo con Lang y Tiede (2003), esta extensión ofrece un conjunto seleccionado de métricas para cubrir investigaciones ecológicas relacionadas con la estructura básica del paisaje y son organizadas de acuerdo con los principales aspectos de análisis en patrones estructurales de los paisajes.

Antes de procesar las métricas para los fragmentos de la silvicultura mapeados en la zona de estudio, se calculó en ambiente SIG las áreas en hectáreas de cada fragmento, luego fueron agrupadas en 5 clases: clase 1 ( 0 a 1 ha) muy pequeñas, clase 2 ( 1 a 10 ha) pequeñas; clase 3 (10 a 50 ha) medianas, clase 4 (50 a 100 ha) grandes y clase 5 (100 a 500 ha) muy grandes, con la finalidad de un mejor análisis de la espacialización y tamaño de los fragmentos.

Para la obtención de los trechos superior, medio e inferior del área de estudio fue propuesta una segmentación de la cuenca hidrográfica en función del perfil longitudinal del rio Yaguarón, por medio de la metodología propuesta por Guedes et al. (2007), donde se cruzaron los valores de altitud versus distancia para generar una curva de mejor ajuste resultando en una función logarítmica. De esta forma los pontos de interceptación de la curva del perfil longitudinal con la curva de mejor ajuste proporcionaron cada uno de los trechos del rio Yaguarón, y así sobre estos segmentos analizar la distribución de los fragmentos de la silvicultura por medio de las métricas descritas en Cuadro 1. 
Cuadro 1: Índices de ecología del paisaje y descripción de las métricas calculadas por el V-Late.

\begin{tabular}{|c|c|c|c|c|}
\hline Grupo & Sigla & Métrica & Unidades & Descripción \\
\hline \multirow{2}{*}{ 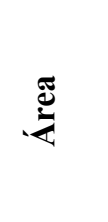 } & $\mathrm{CA}$ & Área da clase & ha & $\begin{array}{l}\text { Suma de las áreas de todas las manchas. Mayores valores } \\
\text { indican dominancia en la matriz del paisaje. }\end{array}$ \\
\hline & PP & Porcentaje del paisaje & $\%$ & $\begin{array}{l}\text { Cuantifica el porcentaje de cobertura con relación al total } \\
\text { del paisaje. }\end{array}$ \\
\hline \multirow{3}{*}{ 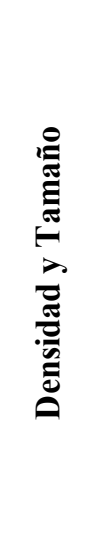 } & NP & Número de manchas & Adimensional & $\begin{array}{l}\text { Número total de manchas. Mayor valor representa } \\
\text { fragmentación del paisaje y valor menor, adhesión o } \\
\text { extinción de fragmentos de la misma clase. }\end{array}$ \\
\hline & MPS & $\begin{array}{l}\text { Tamaño médio de la } \\
\text { mancha }\end{array}$ & ha & $\begin{array}{l}\text { Revela grado de fragmentación por función del número de } \\
\text { fragmentos y del área total ocupada por determinada } \\
\text { clase. Fragmentos con tamaños mayores representan } \\
\text { mayor diversidad, ya que la riqueza de especies depende } \\
\text { de la dimensión de los fragmentos. }\end{array}$ \\
\hline & PSSD & $\begin{array}{l}\text { Desviación estándar } \\
\text { del tamaño de la } \\
\text { mancha }\end{array}$ & ha & $\begin{array}{l}\text { Razón de variación del tamaño de las manchas. Valores de } \\
\text { área altos, representan fragmentos con tamaño muy } \\
\text { encima de la media, y viceversa. }\end{array}$ \\
\hline \multirow{2}{*}{ 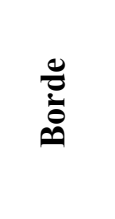 } & TE & Total de bordes & $\mathrm{m}$ & $\begin{array}{l}\text { Suma del perímetro de las manchas. Cuanto más próximo } \\
\text { a cero, más fuerte es el efecto de borde. }\end{array}$ \\
\hline & $\mathrm{ED}$ & Densidad de bordes & $\mathrm{m} / \mathrm{ha}$ & $\begin{array}{l}\text { Longitud total de los bordes, dividido por el área total del } \\
\text { paisaje. Mayor valor exhibe mayor efecto de borde. }\end{array}$ \\
\hline$\underset{3}{3}$ & MSI & Índice de forma & Adimensional & $\begin{array}{l}\text { Es igual a uno cuando todas las manchas son circulares y } \\
\text { aumenta con la irregularidad en la forma de la mancha }\end{array}$ \\
\hline 莺 & MNN & $\begin{array}{l}\text { Distancia media al } \\
\text { vecino más próximo }\end{array}$ & $\mathrm{m}$ & $\begin{array}{l}\text { Muestra el grado de aislamiento de la mancha. Depende } \\
\text { del número, tamaño y distancia entre las otras manchas del } \\
\text { mismo tipo, localizadas dentro de un radio determinado. } \\
\text { Los valores son iguales a cero cuando no existe ninguna } \\
\text { mancha del mismo tipo dentro del radio determinado y } \\
\text { aumenta a medida que crece el número de manchas, y el } \\
\text { tamaño y distancia entre ellas se hacen menores. }\end{array}$ \\
\hline
\end{tabular}

Fuente: Adaptado de Lang y Tiede (2003), Lang y Blaschke (2009), McGarigal y Marks (1995).

\section{RESULTADOS Y DISCUSIONES}

El primer resultado de este trabajo fue la elaboración del mapeo de la silvicultura de pino y eucalipto presente dentro de la cuenca hidrográfica del rio Yaguarón para el año 2013 (Fig. 3), en el cual se obtuvo un total de 6.730 fragmentos mapeados. 
Figura 3: Mapa de la silvicultura en la cuenca hidrográfica del rio Yaguarón.

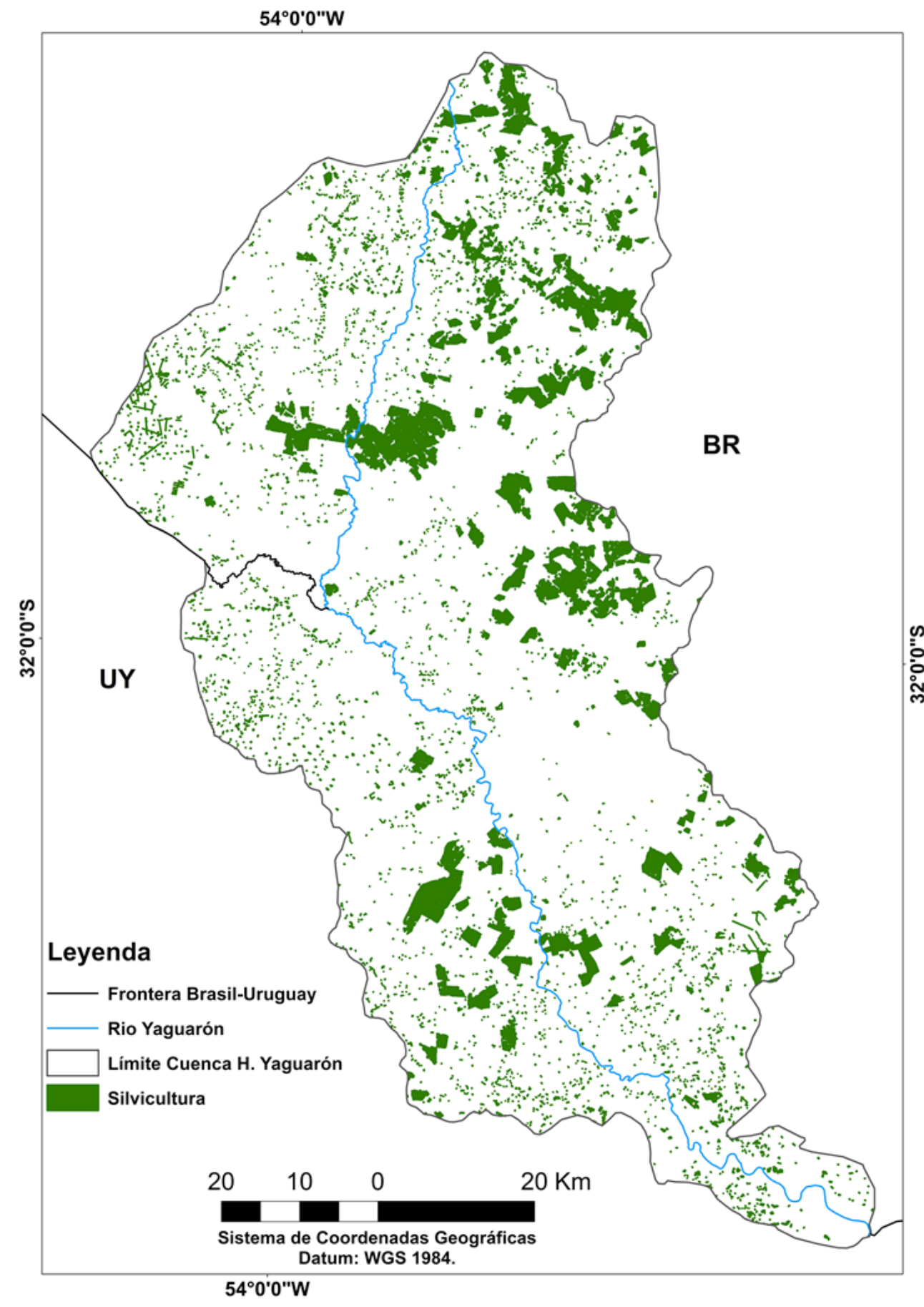

Fuente: autores.

\section{Análisis de los fragmentos de la silvicultura por clases de área}

En cuadro 2 se muestran los cálculos de las métricas del paisaje obtenidos para las clases de los fragmentos de silvicultura presentes dentro de la cuenca hidrográfica del rio Yaguarón. 
Cuadro 2: Métricas calculadas por clases de área.

\begin{tabular}{|c|c|c|c|c|c|c|c|}
\hline \multicolumn{3}{|c|}{ ÍNDICES } & \multicolumn{5}{|c|}{ CLASES DE LOS FRAGMENTOS POR HECTAREAS } \\
\hline GRUPO & MÉTRICAS & SIGLAS & 1 (0 - 1 ha) & 2 ( 1 -10 ha) & 3(10 -50 ha) & $4(50-100 \mathrm{ha})$ & 5 (100 -500 ha) \\
\hline \multirow{2}{*}{ Área } & $\begin{array}{c}\text { Área de la Clase } \\
\text { (ha) }\end{array}$ & $\mathrm{CA}$ & $1.510,9$ & $5.492,4$ & $14.608,2$ & $8.112,3$ & $10.097,0$ \\
\hline & $\begin{array}{c}\text { Porcentaje del } \\
\text { paisaje }\end{array}$ & PP & 3,8 & 13,8 & 36,7 & 20,4 & 25,4 \\
\hline \multirow{3}{*}{$\begin{array}{c}\text { Densidad y } \\
\text { Tamanho }\end{array}$} & $\begin{array}{c}\text { Número de } \\
\text { manchas }\end{array}$ & NP & 4.312 & 1.622 & 624 & 116 & 56 \\
\hline & $\begin{array}{c}\text { Tamaño médio } \\
\text { de la mancha } \\
\text { (ha) }\end{array}$ & MPS & 0,35 & 3,39 & 23,41 & 69,93 & 180,30 \\
\hline & $\begin{array}{c}\text { Desviación típica } \\
\text { del tamaño } \\
\text { mancha (ha) }\end{array}$ & PSSD & 0,24 & 2,38 & 11,01 & 13,57 & 81,33 \\
\hline \multirow{2}{*}{ Borde } & \begin{tabular}{|c}
$\begin{array}{c}\text { Total de bordes } \\
\text { (m) }\end{array}$ \\
\end{tabular} & $\mathrm{TE}$ & $1.194 .260,9$ & $1.615 .053,5$ & 2.101.263,7 & $918.223,0$ & $964.674,1$ \\
\hline & $\begin{array}{c}\text { Densidad de } \\
\text { bordes (m/ha) }\end{array}$ & $\mathrm{ED}$ & 790,63 & 294,14 & 143,84 & 113,19 & 95,54 \\
\hline Forma & $\begin{array}{c}\text { Índice de forma } \\
\text { medio }\end{array}$ & MSI & 1,40 & 1,60 & 1,98 & 2,67 & 3,60 \\
\hline Proximidad & $\begin{array}{l}\text { Distancia média } \\
\text { al vecino más } \\
\text { próximo }(\mathrm{m})\end{array}$ & MNN & 1,4 & 72,7 & $1.716,5$ & 940,5 & $1.319,3$ \\
\hline
\end{tabular}

Fuente: autores.

Los resultados obtenidos de las métricas calculadas para los índices de área, densidad y tamaño, destacan que en número absoluto (NP) las clases 1 y 2 exponen la mayor cantidad de polígonos totalizando 5.934 fragmentos mapeados, que representan el 17,6\% del área ocupada en la cuenca hidrográfica por ambas clases, siendo que las clases 3, 4 y 5 con polígonos de áreas mayores a 10 hectáreas, representan más del $80 \%$ del área total de la silvicultura concentrados en menor número de fragmentos como se puede observar en la Figura 4.

Figura 4: Número de fragmentos por clases (Esq.) y p porcentaje del área de ocupación en el paisaje por clases (Der.)
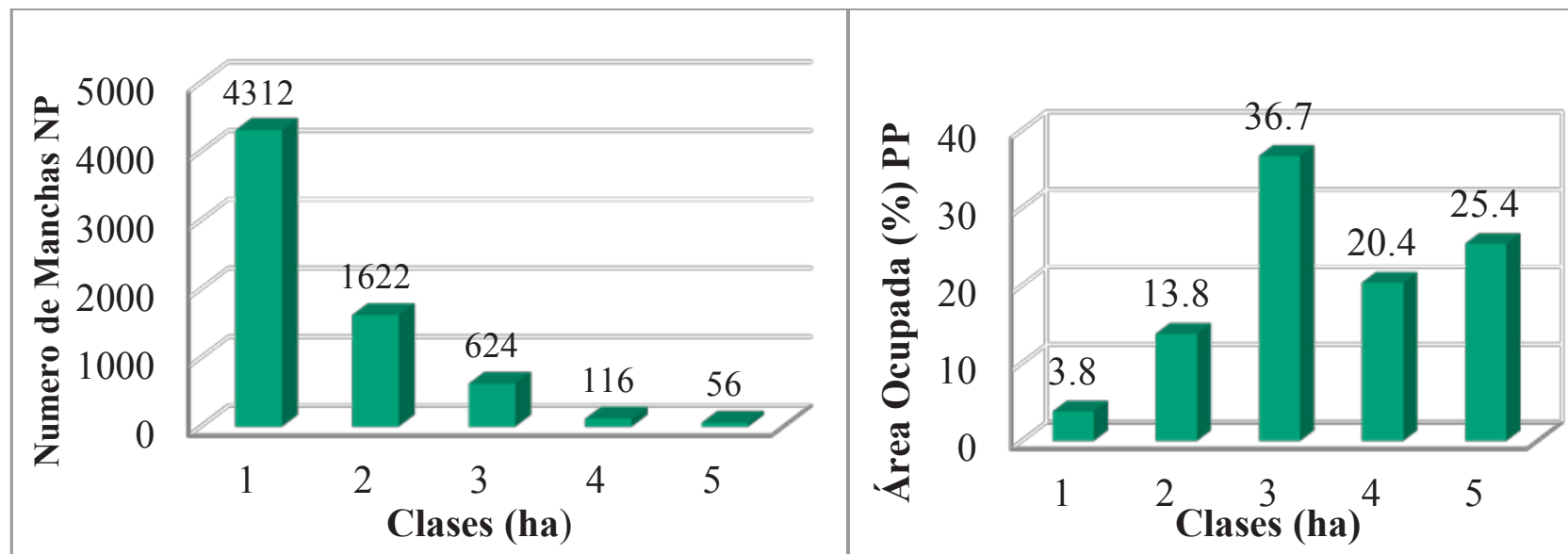

Fuente: autores. 
De esta forma se infiere que las clases 1 y 2 corresponden a pequeñas plantaciones que no fueron destinadas para ocupar grandes áreas, obedeciendo a procesos culturales que reflejan el uso tradicional de los árboles de eucalipto y pino con funciones de corta viento, cercas vivas o pequeñas plantaciones usadas como abrigo para el ganado y para obtención de leña usada para uso doméstico en las estancias (AB'SÁBER, 2005; BIKOWSKI, 2009). Las clases 3, 4 y 5 exhiben mayor ocupación por cuenta de fragmentos de tamaño medio a muy grandes, indicando proceso de expansión de la silvicultura de pino y eucalipto dentro de la zona de estudio por cuenta de las empresas forestales, con el objetivo de obtener madera para la producción de pasta de celulosa y papel, satisfaciendo así las demandas mundiales de estos productos, siendo que el aumento productivo de esta actividad es incentivado por políticas publicas tanto en el Uruguay como en el Brasil, para dinamizar la economía de la región (FREITAS, 2004; BINKOWSKI, 2009)

Entre los índices de borde, la métrica de densidad de borde (ED) que es inversamente proporcional al área ocupada por cada clase, señala un mayor efecto de borde para las clases 1 y 2 con valores de 790,63 m/ ha y 294,14 m/ ha respectivamente, representando mayor fragmentación, así las clases 3, 4 y 5 con valores menores 143,84 m/ha; 13,19 m/ ha y $95,54 \mathrm{~m} /$ ha respectivamente, apuntan a un bajo efecto de borde.

En términos ecológicos los fragmentos de grandes áreas se consideran más conservados (McGARIGAL; CUSHMAN; REGAN, 2005), sin embargo al ser la silvicultura una actividad antrópica, las áreas menores que presentan mayor densidad de borde y que corresponden a cercas vivas, corta vientos o pequeñas plantaciones con características funcionales anteriormente descritas, permanecen en el paisaje durante más tiempo, siendo ambientalmente más estables e integrándose en el paisaje, mientras que las áreas mayores que representan menor fragmentación, son cultivos comerciales para abastecimiento industrial, que permanecen en el paisaje por periodos cortos de tiempo alternándose en ciclos productivos de plantación, crecimiento y cosecha, lo que según Lima (1993) y Da Silva, (2012) traen para el paisaje procesos de alteración, y momentos de inestabilidad.

\section{Análisis de la silvicultura en relación a las clases de vegetación natural}

En esta etapa del trabajo se observó que la fisionomía vegetal predominante en el área de estudio, es la vegetación tipo Estepe con 577.925 hectáreas ocupando el 78\% del área total, presentando esta clase como elemento matriz del paisaje de la cuenca hidrográfica. En secuencia se encuentran la Floresta Estacional Decidual con 70.985 hectáreas (9,6\%), la silvicultura con 39.818 hectáreas (5,4\%), Savanas Estépicas 35.720 (4,8\%) y Áreas de Formações Pioneiras con 9.568 (1,3\%). En total se contabilizaron 8.828 polígonos ocupando un área de 734.015 hectáreas dentro de la cuenca hidrográfica del rio Yaguarón.

El cuadro 3 presenta los índices de ecología del paisaje obtenidos para las clases de vegetación de la cuenca hidrográfica del rio Yaguarón. 
Cuadro 3: Índices del paisaje por clases de vegetación natural presente en la cuenca del rio Yaguarón.

\begin{tabular}{|c|c|c|c|c|c|c|c|}
\hline \multicolumn{3}{|c|}{ ÍNDICES } & \multicolumn{5}{|c|}{ CLASES DEL PAISAJE } \\
\hline GRUPO & METRICAS & SIGLAS & Silvicultura & $\begin{array}{l}\text { Áreas das } \\
\text { Formaçõe } \\
\text { Pioneiras } \\
\end{array}$ & Estepe & \begin{tabular}{|c} 
Floresta \\
Estacional \\
Decidual \\
\end{tabular} & $\begin{array}{l}\text { Savanas } \\
\text { Estépica }\end{array}$ \\
\hline Área & $\begin{array}{c}\text { Área de la } \\
\text { Clase/tamaño (ha) }\end{array}$ & CA & 39.819 & 9.568 & $577.925,3$ & $70.984,5$ & 35.719 \\
\hline \multirow{3}{*}{$\begin{array}{l}\text { Densidad y } \\
\text { Tamaño }\end{array}$} & Número de manchas & NP & 6.730 & 23 & 1.279 & 611 & 185 \\
\hline & $\begin{array}{c}\text { Tamaño medio de la } \\
\text { mancha (ha) }\end{array}$ & MPS & 5,9 & 416,0 & 451,9 & 116,2 & 193,1 \\
\hline & $\begin{array}{c}\text { Desviación estándar } \\
\text { del tamaño mancha } \\
\text { (ha) }\end{array}$ & PSSD & 21,1 & 1.134 & 5.401 & 353,1 & 804,6 \\
\hline \multirow{2}{*}{ Borda } & Total de bordes (m) & $\mathrm{TE}$ & $6.793 .375,1$ & $273.039,8$ & $20.393 .012,8$ & 5.629.761,4 & $2.353 .866,5$ \\
\hline & $\begin{array}{l}\text { Densidad de bordes } \\
\qquad(\mathrm{m} / \mathrm{ha})\end{array}$ & ED & 170,57 & 28,37 & 35,39 & 79,29 & 66,02 \\
\hline Forma & $\begin{array}{l}\text { Índice de forma } \\
\text { medio }\end{array}$ & MSI & 1,54 & 2,02 & 2,26 & 2,80 & 2,66 \\
\hline Proximidad & $\begin{array}{l}\text { Distancia media al } \\
\text { vecino más próximo } \\
(\mathrm{m})\end{array}$ & MNN & 541,3 & 306,8 & $43.088,3$ & $9.324,7$ & $1.359,3$ \\
\hline
\end{tabular}

Fuente: autores.

Según Sousa (2011) el tamaño medio de la mancha es considerado un buen indicativo de grado de fragmentación en función del número de fragmentos y del área total ocupada por las clases. Siguiendo este parámetro las métricas calculadas para las clases de vegetación apuntan un numero de manchas (NP) predominante para la clase Silvicultura (6.730 fragmentos), correspondiendo a un tamaño medio de manchas (MPS) de 5,9 hectáreas; si tenemos en cuanta el área total (CA) de 39.818 hectáreas, el tamaño medio de la mancha (MPS) de la silvicultura presenta un valor bajo, lo que indica mayor presencia de fragmentos pequeños de esa clase.

Para las clases Áreas de Formações Pioneiras y Savanas Estépicas el número de fragmentos (NP) en relación con el área de la clase (CA), son valores bajos y con valores del tamaño medio de la mancha (MPS) altos, señalando un menor grado de fragmentación.

La Desviación estándar del tamaño mancha (ha) muestra que la menor correlación de la clase silvicultura es con la clase Estepe, siendo un indicativo de integración de esta clase en relación con la matriz del paisaje.

La métrica de Densidad de borde (ED) muestra a la silvicultura como la clase más fragmentada seguida de la Floresta Estacional Decidual. Según Santos e Rocha (2015) los bordes de los fragmentos de áreas menores son locales susceptibles a cambios en sus ambientes de transición.

El índice de forma medio revela que las formas de las clases de vegetación natural de la cuenca hidrográfica, son en general irregulares con formatos más complejos al presentar valores encima de dos, volviéndolas clases más vulnerables a sufrir efectos de borde. La 
clase silvicultura presenta formatos más simples con tendencia a volverse irregulares, sin embargo sus formas obedecen a técnicas de cultivos y no a una distribución natural.

La métrica de distancia media al vecino más próximo, muestra la clase Estepe (en ausencia de usos de suelo en la cuenca hidrográfica) con mayor grado de conectividad en el área de estudio, las clases Formações Pioneiras, Silvicultura y Savanas Estépicas son las clases con mayor grado de aislamiento. El aislamiento de la clase de Formações Pioneiras se debe a que es una vegetación de ocupación primaria de carácter edáfico tendiente a desarrollarse a lo largo de planicies fluviales del rio Yaguarón y alrededor de las depresiones aluviales de la laguna Merín (IBGE, 1992; MARCHESI; FAROPPA; ANNALA, 2005).

La clase silvicultura se presenta aislada debido a su naturaleza antrópica ya que en el área de estudio se cultiva como porción de forestas aisladas en medio del campo para dar sombra al ganado, además las estancias se encuentran relativamente lejos las unas de las otras lo que aumenta el grado de aislamiento de la clase, también puede ser resultado del propio proceso de implantación de la silvicultura en la región, ya que esta clase está condicionada por técnicas de cultivo, factores climáticos, relieves y suelos (CAL et al., 2008; WILCKEN et al., 2008; FLORES; FILIPPINI ALBA; WREGE, 2009).

\section{Análisis de la silvicultura con relación a los trechos de la cuenca hidrográfica}

En la Figura 5 se puede observar la relación de los valores de altitud versus distancia que formaron el perfil longitudinal del rio Yaguarón, sobre este se generó una curva de mejor ajuste (línea roja) resultado de una función logarítmica. Los puntos de interceptación del perfil longitudinal con la curva de mejor ajuste fueron definidos como los trechos principales de la cuenca hidrográfica.

Figura 5: Tramos principales de la cuenca hidrográfica del rio Yaguarón, en función al perfil longitudinal.

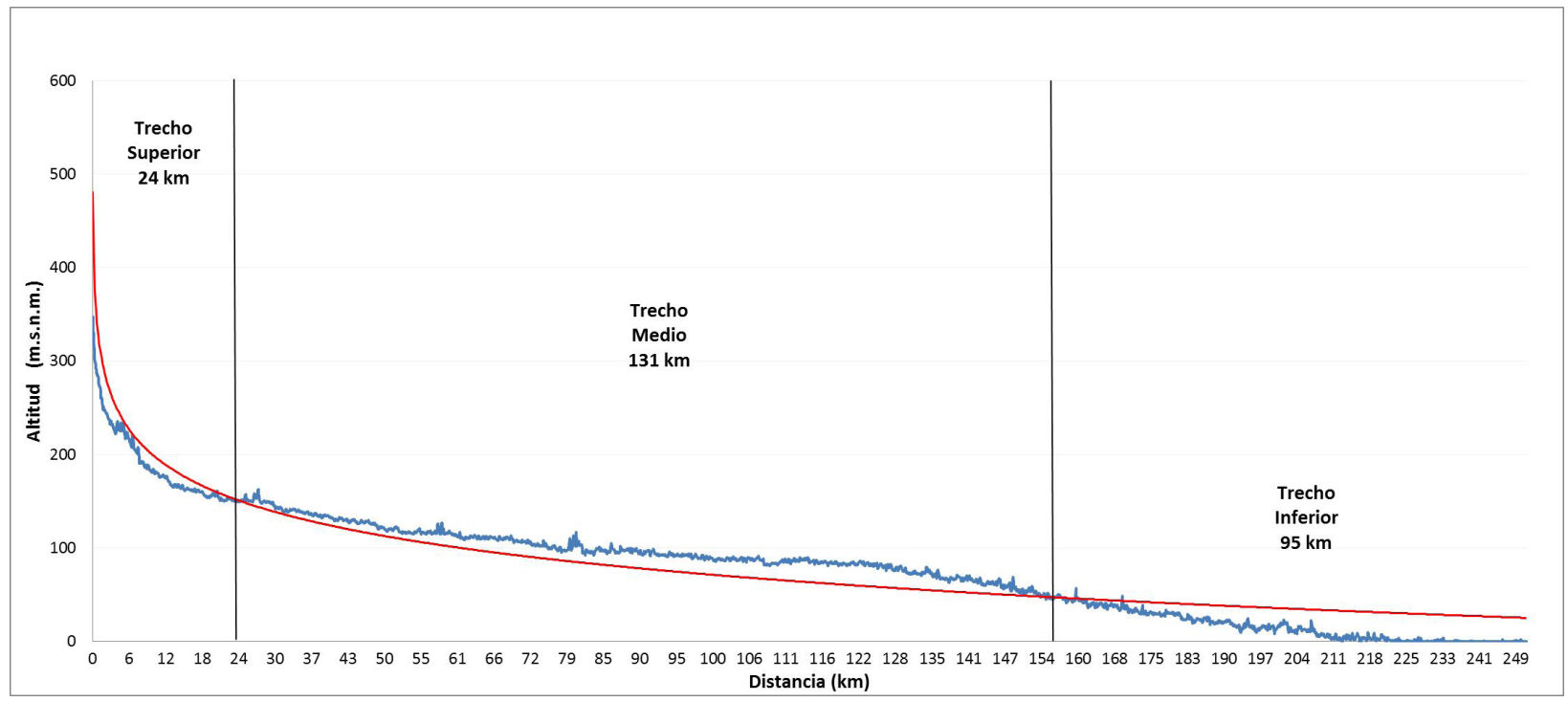

Fuente: autores. 
El trechos superior posee en distancia $24 \mathrm{~km}$ con un área de $679 \mathrm{~km}^{2}$, en este trecho las elevaciones van desde 150 a 350 metros sobre el nivel del mar (m.s.n.m) en este se localiza el nacimiento principal del rio Yaguarón y de uno de los principales afluentes de la cuenca hidrográfica, el arroyo Candiota (FEPAM, 2013).

La distancia del trecho medio es de $131 \mathrm{~km}$ con un área de $5.520 \mathrm{~km}^{2}$. Prevalecen las elevaciones desde los 50 hasta los 200 m.s.n.m y se caracteriza por sus relieves suaves ondulados con pocos afloramientos rocosos y poca presencia de área de bañados (SOMBROEK et al., 1970; ACHKAR; DOMINGUEZ; PESCE, 2012).

El trecho inferior cuenta con una distancia de $95 \mathrm{~km}$, su área total es de $1.207 \mathrm{~km}^{2}$. Predominan los relieves más bajos de la cuenca hidrográfica con elevaciones entre los $0 \mathrm{a}$ 50 m.s.n.m. Este trecho contiene las áreas de formaciones pioneras siendo áreas de planicies entre altas y bajas, además de áreas de bañados fluviales (SOARES, 2009; ACHKAR; DOMINGUEZ; PESCE, 2012).

Los resultados de las métricas de área, densidad y tamaño calculadas por clases de fragmentos para cada trecho de la cuenca hidrográfica son presentados en Cuadro 4.

Cuadro 4: Cálculo de las métricas del paisaje para la silvicultura en diferentes trechos de la cuenca.

\begin{tabular}{|c|c|c|c|c|c|c|c|c|}
\hline \multicolumn{4}{|c|}{ ÍNDICES } & \multicolumn{5}{|c|}{ CLASES DE LOS FRAGMENTOS DE SILVICULTURA } \\
\hline GRUPO & MÉTRICAS & SIGLAS & TRECHOS & 1. (0 a 1$)$ & 2. $\left(\begin{array}{l}1 \\
\text { a }\end{array} 10\right)$ & 3. $(10$ a 50$)$ & 4. $(50$ a 100$)$ & 5. $(100$ a 500$)$ \\
\hline \multirow{3}{*}{ Área } & \multirow{3}{*}{$\begin{array}{c}\text { Área de la } \\
\text { Clase/tamaño (ha) }\end{array}$} & \multirow{3}{*}{$\mathrm{CA}$} & Superior & 164,7 & 864,3 & $2.280,2$ & $1.157,4$ & $1.594,4$ \\
\hline & & & Medio & 981,9 & $3.862,8$ & $11.081,2$ & $6.772,0$ & $6.271,7$ \\
\hline & & & Inferior & 325,8 & 732,3 & $1.280,9$ & 262,3 & $2.187,5$ \\
\hline \multirow{9}{*}{$\begin{array}{l}\text { Densidad y } \\
\text { Tamaño }\end{array}$} & \multirow{3}{*}{ Número de manchas } & \multirow{3}{*}{ NP } & Superior & 451 & 244 & 97 & 16 & 9 \\
\hline & & & Medio & 2.944 & 1.125 & 469 & 95 & 34 \\
\hline & & & Inferior & 928 & 249 & 56 & 3 & 10 \\
\hline & \multirow{3}{*}{$\begin{array}{l}\text { Tamaño } \\
\text { médio de la mancha } \\
\text { (ha) }\end{array}$} & \multirow{3}{*}{ MPS } & Superior & 0,39 & 3,57 & 23,41 & 68,93 & 160,88 \\
\hline & & & Medio & 0,34 & 3,44 & 23,61 & 69,96 & 179,61 \\
\hline & & & Inferior & 0,37 & 2,98 & 22,33 & 69,20 & 200,18 \\
\hline & \multirow{3}{*}{$\begin{array}{l}\text { Desviación estándar } \\
\text { del tamaño mancha } \\
\text { (ha) }\end{array}$} & \multirow{3}{*}{ PSSD } & Superior & 0,24 & 2,38 & 10,79 & 12,91 & 60,26 \\
\hline & & & Medio & 0,24 & 2,43 & 11,13 & 13,77 & 88,95 \\
\hline & & & Inferior & 0,22 & 2,07 & 10,53 & 8,62 & 66,80 \\
\hline
\end{tabular}

Fuente: autores.

En la figura 6 se puede observar que el mayor número de fragmentos de silvicultura (NP) y las mayores áreas ocupadas (CA) por los cultivos, están presentes en el trecho medio para todas las clases. 
Figura 6: Número de manchas para cada clase (Esq.) y área de cada clase (Der.) en los diferentes tramos
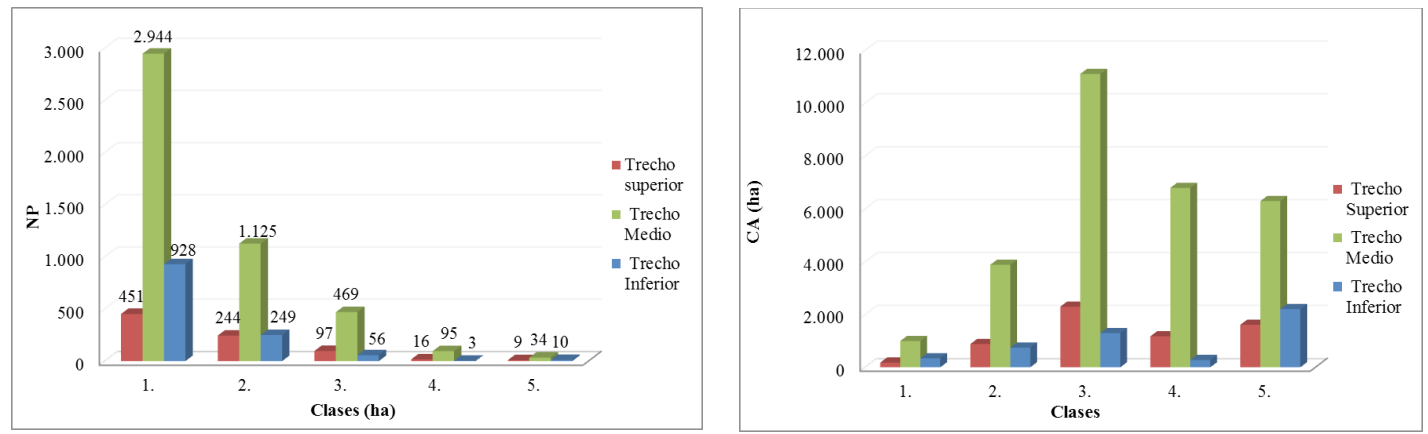

Fuente: autores.

El hecho de que el trecho medio contenga el mayor número de manchas y mayores áreas se debe a que espacialmente es más grande que los otros trechos de la cuenca hidrográfica, también a que sus relieves son planos o suave ondulados cubiertos principalmente por pastos, con poca presencia de bañados, lo que según Flores, Filippini y Wrege (2009) hace de esa zona las más aptas para implementar la silvicultura. También en Uruguay el área espacial que corresponde al trecho medio de esta cuenca hidrográfica, fue considerada por la Comisión Nacional de Estudios Agroeconómicos de la Tierra CONEAT, como aptas para la producción de pastos para el ganado de corte y de prioridad forestal para producción de pasta de celulosa (CAL et al., 2008)

También se puede observar en la figura 6 que en los trechos superior e inferior, la cantidad de áreas plantadas de árboles de pinos y eucaliptos no son tan significativas.

En el trecho superior predominan los fragmentos de silvicultura de las clases 3 y 4 (tamaños medios entre 10 a 100 hectáreas) mostrando tendencia de transición hacia grandes cultivos comerciales de la clase 5. En este trecho el relieve rugoso de la cuenca hidrográfica es un limitante para la silvicultura, ya que esta sobre el borde sur del escudo Sul Riograndense, con predominio de suelos litólicos, mal o imperfectamente drenados y de fertilidad baja (FLORES; FILIPPINI ALBA; WREGE 2009).

Las métricas mostraron para el trecho inferior un aumento de área en los fragmentos de la clase 5 (100 a 500 ha), sin embargo estas áreas están concentradas en solo 10 fragmentos de silvicultura, no obstante se tiene un número significativo de fragmentos pequeños que cumplen funciones de corta vientos. La ausencia de cultivos comerciales en este trecho se debe a la gran cantidad de áreas húmedas haciéndolo poco favorable para la silvicultura a gran escala ya que el exceso de agua puede generar problemas fitosanitarios en las plantaciones (LOPES et al., 2005) además estas áreas por sus sustratos edáficos son priorizadas para el cultivo de arroz (SOMBROEK et al., 1970; STEINKE; SAITO 2008; ACHKAR; DOMINGUEZ; PESCE, 2012; SERRENTINO B., 2013). 


\section{CONCLUSIONES}

Los resultados de este trabajo mostraron que la silvicultura se presenta de forma homogénea dentro de la cuenca hidrográfica, siendo que la mayor ocupación se da por cuenta de los fragmentos pequeños, corroborando las características tradicionales de corta vientos o cercas vivas de los árboles de eucaliptos y pinos en la cuenca hidrográfica. Los pocos fragmentos de las clases medias se mostraron como "clase de transición" entre pequeños bosques a cultivos comerciales y los fragmentos de la clase grande como elementos divergentes del paisaje.

Siendo la silvicultura la más significativa clase antrópica de uso de suelo dentro de un paisaje de vegetación natural en la cuenca hidrográfica del rio Yaguarón, es normal percibirla como causante de fragmentación en el paisaje, sin embargo en este escenario específico los impactos de la silvicultura son relativamente equilibrados. La fragmentación es mayor en términos de polígonos existentes en algunas clases, pero en términos de impacto estos serían menores, ya que las áreas de silvicultura en esta cuenca son en general pequeñas plantaciones aisladas en medio del campo no mayores a $3 \mathrm{ha}$, siendo pocos los cultivos implementados para producción maderera a grade escala, teniendo funciones menos impactante en el paisaje del área de estudio y mostrándose en equilibro.

\section{REFERENCIAS}

AB'SÁBER, A.N. Os domínios de natureza no Brasil: potencialidades paisagísticas. 3.ed. São Paulo: Ateliê Editorial, 2005.

ACHKAR, M.; DOMINGUEZ A.; PESCE F. Cuenca de la Laguna Merín-Uruguay. Aportes para la discusión ciudadana. Montevideo: IECA, 2012. Programa Uruguay Sustentable/REDES-AT.

AYAD, Y. Assessment of landscape ecological metrics: shape complexity and fragmentation of the abandoned strip mine patches in Toby Creek watershed. In: ANNUAL ESRI INTERNATIONAL USER CONFERENCE, 25, 2005, San Diego.

BINKOWSKI, P. Conflitos ambientais e significados sociais em torno da expansão da silvicultura de eucalipto na "metade sul" do Rio Grande do Sul. 2009. Dissertação (Mestrado em Desenvolvimento Rural) - Universidade Federal do Rio Grande do Sul, UFRGS, Porto Alegre - RS.

CAL, A. et al. Mapa de Cobertura del Suelo de Uruguay. Land Cover Classification System. Montevideo, 2008. Disponible en: <http://web.renare.gub.uy/media/cobertura/informes/Cobertura2008.pdf>. Acceso en: 20 jun. 2017.

CHELOTTI, M.C. Reterritorialização e identidade territorial. Revista Sociedade \& Natureza, v. 22, n. 1, p. 165-180, 2010.

CLEMENTE BATALLA, G.I.Sociedades Fronterizas, Actores Sociales y Políticas de Ordenamiento Territorial en la Frontera Uruguay-Brasil. In: SEMINARIO DA RED IBEROAMERICANA DE INVESTIGADORES SOBRE GLOBALIZACIÓN Y TERRITORIO (RII), 13, 2014, Salvador. Disponible en: http:/ /www.rii.sei. ba.gov.br/anais-do-xiii-seminario-rii/. Acceso en: 14 jul. 2017.

CRACCO, M. et al. Importancia Global de la Biodiversidad de Uruguay. S.l: S.ed., 2005. Serie Documentos de Trabajo. 
CRM. Mina Candiota. Governo do Estado de Rio Grande do Sul. Secretaria de Minas e Energia. Disponible en: <http://www.crm.rs.gov.br/conteudo/858/?Mina-de-Candiota\#.VbLxPvlViko>. Acceso en: 30 mayo 2017.

DA SILVA, M.D. Os cultivos florestais do pampa, no sul do Rio Grande do Sul: desafios, perdas e perspectivas frente ao avanço de novas fronteiras agrícolas. Revista Floresta, v. 42, n. 1, p. 215-226, 2012.

DIAZ, D; TESÓN, N.; GARCÍA, M.A. Efectos Ambientales de las Forestaciones de Eucaliptos en el Noreste de Entre Ríos. In: JORNADAS FORESTALES DE ENTRE RÍOS, 21, 2006, Concordia. Anais ... 15p.

FARINA, A. Principles and Methods in Landscape Ecology: towards a science of the landscape. S.l.: Springer Science \& Business Media, 2008. v.3.

FEPAM. L60 Bacia Hidrográfica do Rio Jaguarão. 2012. Disponible en: http://www.fepam.rs.gov.br/ qualidade/bacia_jaguarao.asp. Acceso en: 14 jul. 2017.

FLORES, C.A.; FILIPPINI ALBA, J.M.; WREGE, M.S. Zoneamento agroclimático do eucalipto para o Estado do Rio Grande do Sul e Edafoclimático na Região do Corede Sul - RS. Pelotas: Embrapa Clima Temperado, 2009.

FORMAN, R.T. Some general principles of landscape and regional ecology. Landscape Ecology, v. 10, n. 3, p. 133-142, 1995.

.; GODRON, M. Patches and structural components for a landscape ecology. BioScience, v. 31, n. 10, p.733-740, 1981.

FREITAS, M. et al. Mapa hidrogeológico do Rio Grande do Sul: um avanço no conhecimento das águas subterrâneas no estado. In: CONGRESSO BRASILEIRO DE ÁGUAS SUBTERRÂNEAS, 13, 2004, Cuiabá.

GUEDES, I.C et al. Análise de perfis longitudinais de drenagens da Bacia do Rio Santo Anastácio (SP) para deteç̧ão de possíveis deformações neotectônicas. Revista Geociências, v. 5, n. 1, p. 75-102, 2007.

HASENACK, H.; CORDEIRO, J.L.P. Mapeamento da cobertura vegetal do Bioma Pampa. Porto Alegre, UFRGS Centro de Ecologia. Relatório Técnico Ministério do Meio Ambiente: Secretaria de Biodiversidade e Florestas no Âmbito do Mapeamento da Cobertura Vegetal dos Biomas Brasileiros. Porto Alegre: UFRGS, 2006.

IBGE. Manual técnico da vegetação brasileira. Rio de Janeiro, 1992. Série Manuais Técnicos em Geociências, 1.

IBGE. Produto Interno Bruto dos Municípios 2004-2008. Série Coleção Ibgeana; Contas nacionais. Disponible en: <http:/ / biblioteca.ibge.gov.br/pt/ biblioteca-catalogo?view=detalhes\&id=247236>. Acceso en: 13 mayo 2017.

INE Censo 2011. Departamento de Cerro Largo. Disponible en: http://www.ine.gub.uy/censos2011/ resultadosfinales/cerrolargo.html> Acceso en: 20 set. 2017.

IRASTORZA VACA, P. Integración de la ecología del paisaje en la planificación territorial: Aplicación a la comunidad de Madrid. 2006. Tesis (Doutorado) - Universidad Politecnica de Madrid.

LANG, S.; BLASCHKE, T. Análise da Paisagem com SIG. São Paulo: Oficina de Textos, 2009.

.; TIEDE, D. V-Late Extension für ArcGIS. Innsbruck: ESRI, 2003. Disponible en: < https://sites. google.com/site/largvlate/gis-tools/v-late>. Acesado en: 18 jul. 2014.

LIMA, W. de P. Impacto Ambiental do Eucalipto. São Paulo: Ed. USP, 1993.

LOPES, J.L.W. et al. Efeitos da irrigação na sobrevivência, transpiração e no teor relative de água na folha em mudas de Eucalyptus grandis em diferentes substratos. Scientia Forestalis, n. 68, p. 97-106, 2005.

McGARIGAL, K.; CUSHMAN, S.; REGAN, C. Quantifying terrestrial habitat loss and fragmentation: a protocol. Amherst: US For. Serv. Gen. Tech. Repl. RM-GTR, 2005.

McGARIGAL, K.; MARKS, B.J. Fragstasts: Spatial pattern analysis program for quantifying landscape structure. Portland (OR): Pacific Northwest Research Station, 1995. Gen. Tech. Rep. PNW-GTR-351 
MARCHESI, E.; FAROPPA, C.; ANNALA, K. Flora y vegetación del Uruguay. Montevideo: C. Faroppa \& K. Annala, 2004. Informe Ambiental Resumen.

MATTEUCCI, S.D.; MATTEUCCI, S.D.; BUZAI, G.D. La cuantificación de la estructura del paisaje. In: .; BUZIA, G.D. (Eds.) Sistemas ambientales complejos: herramientas de análisis espacial. Buenos Aires: EUDEBA, 1998. p. 271-291.

MEENTEMEYER, V.; BOX, E O. Scale effects in landscape studies. In: TURNER, M.G. Landscape heterogeneity and disturbance. New York: Springer, 1987. p. 15-34.

METZGER, J.P. Estrutura da paisagem: o uso adequado de métricas. In: CULLEN JUNIOR, L.; VALLADARES P.C.; RUDRAN, R. (Org). Métodos de estudo em biologia da conservação e manejo da vida silvestre. Curitiba: Ed. UFPR, 2006. p. 423-453.

PEREZ ÁLVAREZ, C. Anisotropía de la susceptibilidad magnética (ASM) de la Formación Sierra de los Ríos (Cerro Largo, Uruguay). 2014. Monografía (Licenciatura en Geología) - Universidad de la Republica, Cerro Largo.

PICKETT, S.T.A.; CADENASSO, M.L. Landscape ecology: spatial heterogeneity in ecological systems. Science, v. 269, n. 5222, p. 331-334, 1995.

RÓTULO, D.; DAMIANI, O. El caso de la integración fronteriza Uruguay Brasil: dimensiones analíticas e hipótesis de trabajo preliminares. Universidad ORT Uruguay, 2010. Documento de Investigación n. 61. Disponible en: http://www.ort.edu.uy/facs/pdf/documentodeinvestigacion61.pdf. Acceso en: 08 oct. 2017.

SERRENTINO B., C.M. Cuenca Binacional de la Laguna Merin. Centro del Agua para América Latina y el Caribe, 2013. Disponible en: http://infoagua.centrodelagua.org/doc/171_File.pdf. Acceso en: 10 oct. 2017.

SOARES, M N. Proposta de zoneamento ecológico econômico para o município de Pedras Altas-RS. 2009. Dissertação (Mestrado em Geografia) - Universidade Federal do Rio Grande, Pelotas - RS.

SOMBROEK, W.G. et al. Estudo dos solos na Bacia da Lagoa Mirim. Apêndice B: descrições, dados físicos e químicos: parte brasileira. Pelotas: CLM/PNUD/FAO, 1970. (Relatório Interno). Tradução de: Soil studies in the Merin Lagoon basin.

STEINKE, V.A.; SAITO, C.H. Exportação de carga poluidora para identificação de áreas úmidas sob risco ambiental na bacia hidrográfica da Lagoa Mirim. Sociedade \& Natureza, v. 20, p. 43-67, 2008.

VERDUM, R.; BASSO, L.A.; SUERTEGARAY, D.M. Rio Grande do Sul: paisagens e territórios em transformação. Porto Alegre: Ed. UFRGS, 2004. v.1.

WILCKEN, C. F. et al. Guia prático de manejo de plantações de eucalipto. São Paulo: FEPAF, 2008.

Data de submissão: 17/08/2017

Data de aceite: 27/10/2017 